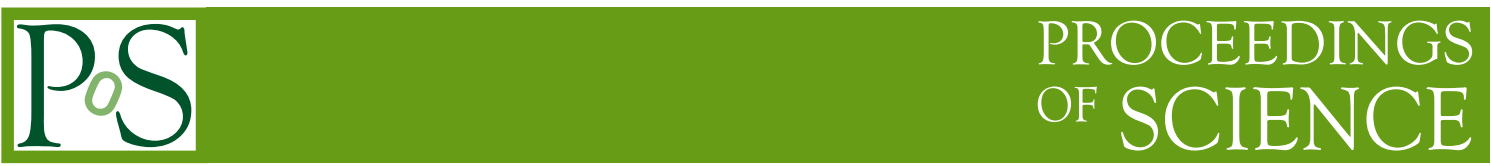

\title{
Continuum Study of Heavy Quark Diffusion
}

\author{
T. Neuhaus ${ }^{* \dagger}$ \\ Institute for Advanced Simulation, FZ Jülich, D-52425 Jülich, Germany \\ E-mail: t.neuhaus@fz-juelich.de
}

\begin{abstract}
We report on a lattice investigation of heavy quark momentum diffusion within the pure SU(3) plasma above the deconfinement transition with the quarks treated to leading order in the heavy mass expansion. We measure the relevant "colour-electric" Euclidean correlator and based on several lattice spacing's perform the continuum extrapolation. This is necessary not only to remove cut-off effects but also the analytic continuation for the extraction of transport coefficients is well-defined only when a continuous function of the Euclidean time variable is available. We pay specific attention to scale setting in $\mathrm{SU}(3)$. In particular we present our determination for the critical temperature $T_{c}=1 /\left(N_{\tau} a\right)$ at values of $N_{\tau} \leq 22$.
\end{abstract}

9th International Workshop on Critical Point and Onset of Deconfinement - CPOD2014,

17-21 November 2014

ZiF (Center of Interdisciplinary Research), University of Bielefeld, Germany

\footnotetext{
* Speaker.

${ }^{\dagger}$ In collaboration with A. Francis, O. Kaczmarek, M. Laine and H. Ohno.
} 


\section{Introduction}

Among the most important quantities playing a role in the theoretical interpretation of current heavy ion collision experiments at RHIC (Brookhaven National Laboratory, USA) and LHC (CERN, Switzerland) are so-called transport coefficients: shear and bulk viscosity's as well as heavy and light quark diffusion coefficients. Because of strong interactions, these quantities need to be determined by non-perturbative numerical lattice Monte Carlo simulations. This task is a hard one, given that numerical simulations are carried out in Euclidean signature, whereas transport coefficients are Minkowskian quantities, necessitating an analytic continuation [1]. Nevertheless, the problem is solvable in principle [2], provided that lattice simulations reach a continuum limit and that short-distance singularities can be subtracted [3]. We present selected results of transport coefficient calculations and scale setting in pure $S U(3)$ gauge theory. Scale setting is involved in in the proper continuum extrapolation of lattice correlation functions.

Most of the results concerning scales and diffusion in the $S U(3)$ plasma have been published in $[4,5]$ or will be presented elsewhere $[6,7]$. Here we specifically supply additional insight in the determination of $T_{c}$, which at finite temperature is the most fundamental physical observable. We leave out our groups high precision studies of the technical lattice scales $r_{0} / a$, the Sommer scale [8] as well as the Wilson flow scale $\sqrt{t_{0}} / a$ [9]. We will however make use of the data in [10] and determine the "amplitude product" $T_{c} \sqrt{t_{0}}$ using $T_{c}$ data and the Lüscher et. al. $t_{0}$ data set.

\section{Colour electric Correlation Function}

Heavy quarks carry a colour charge and, whenever there are gauge fields present, are therefore subject to a coloured Lorentz force. Like with other transport coefficients the corresponding "lowenergy constants" are easiest to define at vanishing three-momentum; then the Lorentz-force is proportional to the electric field strength. This leads to a "colour-electric correlator" [11, 12],

$$
G_{\mathrm{E}}(\tau)=-\frac{1}{3} \sum_{i=1}^{3} \frac{\left\langle\operatorname{Re} \operatorname{Tr}\left[U\left(\frac{1}{T}, \tau\right) g E_{i}(\tau, \overrightarrow{0}) U(\tau, 0) g E_{i}(0, \overrightarrow{0})\right]\right\rangle}{\left\langle\operatorname{Re} \operatorname{Tr}\left[U\left(\frac{1}{T}, 0\right)\right]\right\rangle}
$$

where $g E_{i}$ denotes the colour-electric field, $T$ the temperature, and $U\left(\tau_{2}, \tau_{1}\right)$ a Wilson line in Euclidean time direction. If the corresponding spectral function, $\rho_{\mathrm{E}}$, can be extracted [1], then the "momentum diffusion coefficient", often denoted by $\kappa$, can be obtained from

$$
\kappa=\lim _{\omega \rightarrow 0} \frac{2 T \rho_{\mathrm{E}}(\omega)}{\omega} .
$$

According to non-relativistic linear response relations (valid for $M \gg \pi T$, where $M$ stands for a heavy quark pole mass) the corresponding "diffusion coefficient" is given by $D=2 T^{2} / \kappa$. We determine stochastic estimates for the function $G_{\mathrm{E}}(\tau)$ from large scale Monte Carlo simulations in finite temperature $T \approx 1.45 T_{c}$ pure gauge $S U(3)$ lattice gauge theory with standard Wilson action and for box sizes, that substantially exceed those of earlier simulation results [13]. The largest volume is $V_{\max }=48 \times 192^{3}$ which is simulated with sufficient statistics at $\beta=7.793$. Table 1 shows run parameters with $N_{\text {stat }}$ labeling the number of statistically independent configurations, while $r_{0} T$ 


\begin{tabular}{|c|c|c|c|c|}
\hline$\beta$ & $N_{\tau}$ & $N_{s}$ & $N_{\text {conf }}$ & $r_{0} T$ \\
\hline \hline 6.872 & 16 & 64 & 100 & 1.111 \\
7.192 & 24 & 96 & 160 & 1.077 \\
7.544 & 36 & 144 & 563 & 1.068 \\
7.793 & 48 & 192 & 223 & 1.055 \\
\hline
\end{tabular}

Table 1: A lattice discretized version of the correlator eq. 2.1 is calculated at the given $\beta$ values and box sizes for the finite high temperature $T \approx 1.45 T_{c}$.

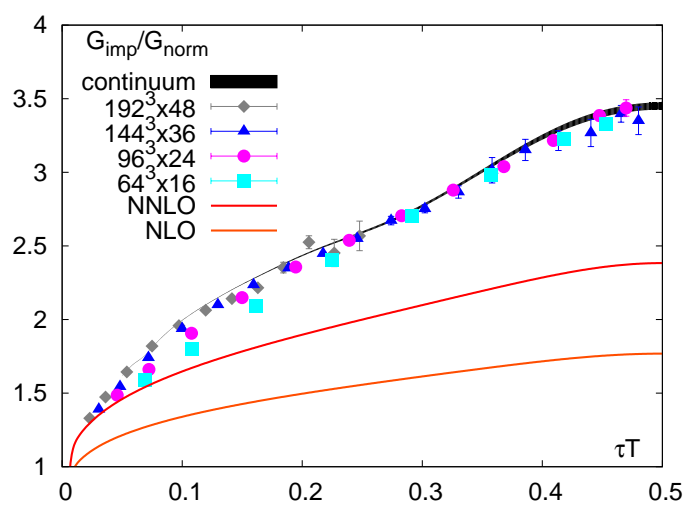

Figure 1: Correlation function ratio $G_{i m p}(\tau T) / G_{\text {norm }}(\tau T)$, see text, with perturbative NLO [19] and NLLO calculations. The black band denotes the desired continuum extrapolation result.

denotes an estimate of the Sommer scale in units of the temporal box extent. We perform quenched QCD calculations for the discrete version of the correlation function $G_{E}(\tau)$. Due to the bad signal to noise ratio of the purely gluonic operator and the rapid decrease of the correlation function with $\tau$ we have to use noise reduction techniques i.e., multi-level updates $[14,15]$ and link-integration "PPR" [16, 17]. Furthermore we use a tree-level improvement [18] to reduce cut-off effects and a NLO perturbative renormalization factor $Z_{\text {pert }}(\beta)$. In Fig. 1 the lattice results for $G_{\text {imp }}(\tau T)$ are shown, normalized to

$$
G_{\text {norm }}(\tau T) \equiv \pi^{2} T^{4}\left[\frac{\cos ^{2}(\pi \tau T)}{\sin ^{4}(\pi \tau T)}+\frac{1}{3 \sin ^{2}(\pi \tau T)}\right] .
$$

Although cut-off effects are visible at small separations and the results become more noisy at large distances on the finer lattices, the results on the four lattices allow for a controlled continuum extrapolation down to distances around $\tau T \sim 0.05$. To obtain the continuum estimate of the correlation function, we perform b-spline or polynomial interpolations for each lattice and at fixed $\tau T$ extrapolate the correlator in $1 / N_{\tau}^{2}$. To allow for needed contributions to the spectral function $\rho_{\mathrm{E}}(\omega)$ we use the Ansatz

$$
\rho_{\text {model }}(\omega)=\max \left\{A \rho_{N N L O}(\omega)+B \omega^{3}, \frac{\omega \kappa}{2 T}\right\},
$$

containing three parameters $A, B$ and $\kappa$, and fit the Euclidean correlator with the form

$$
G_{\text {model }}(\tau)=\int_{0}^{\infty} \frac{\mathrm{d} \omega}{\pi} \rho_{\text {model }}(\omega) \frac{\cosh \left(\frac{1}{2}-\tau T\right) \frac{\omega}{T}}{\sinh \frac{\omega}{2 T}}
$$




\begin{tabular}{|c|c|}
\hline$N_{\tau}$ & $\beta_{c}$ \\
\hline 4 & $5.69275(28)$ \\
6 & $5.89425(29)$ \\
8 & $6.06239(38)$ \\
10 & $6.20872(47)$ \\
12 & $6.33514(45)$ \\
14 & $6.4473(18)$ \\
16 & $6.5457(40)$ \\
18 & $6.6331(20)$ \\
20 & $6.7132(26)$ \\
22 & $6.7986(65)$ \\
\hline
\end{tabular}

Table 2: Numerical $\beta_{c}$ values for pure gauge $S U(3)$ theory with Wilson action.

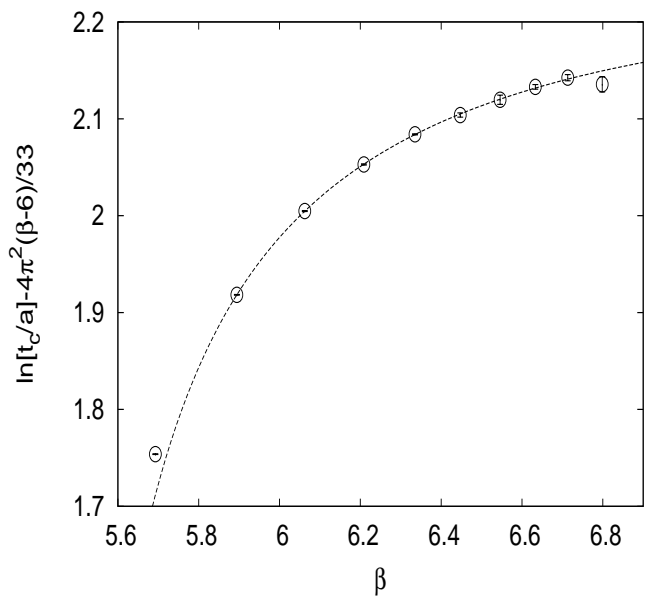

Figure 2: Given the critical time extent $t_{c} / a=N_{\tau}$ as calculated from Table 2 we display the quantity $\ln \left(t_{c} / a\right)-4 \pi^{2}(\beta-6) / 33$ as a function of $\beta$. The curve for $\beta>5.8\left(N_{\tau}>4\right)$ displays the fitted rational Ansatz eq. 3.1, see the text.

in the $\tau T$ range [0.1:0.5] to the continuum. Under the given procedure we find the momentum diffusion coefficient:

$$
\kappa / T^{3}=2.5(4) \quad T \approx 1.45 T_{c} .
$$

For a more detailed presentation we refer here to [5]. We will have to investigate the stability of the numeric value under various model assumptions for the spectral density.

\section{Deconfinement Position at large temporal Extent}

Using alternate finite size scaling arguments [20] from statistical physics, based on phase weight ratios at first order phase transitions we determine the finite temperature transition couplings $\beta_{c}\left(N_{\tau}\right)$ for a maximal value $N_{\tau}=22$. The numerical findings are contained in Table 2. The $\beta_{c}$ data of Table 2 correspond directly to the circles in Fig. 2 where the temporal time extent $t_{c} / a=N_{\tau}$ in units of $a$ at the $S U(3)$ Deconfinement phase transition is suitably logarithmized and 


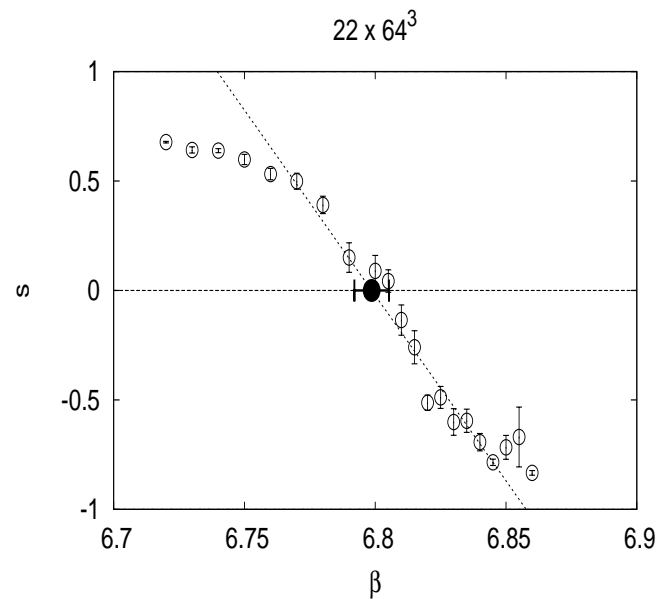

Figure 3: For the $N_{\tau}=22$ and $N_{s}=64$ box we display as a function of $\beta$ the step function $s(\beta)$, whose zero determines the critical point at $N_{\tau}=22$ as in Table 2 . The zero is marked by a solid circle and horizontal error bar and is obtained by the straight line fit, the line in the figure.

subtracted: $\ln \left(t_{c} / a\right)-4 \pi^{2}(\beta-6) / 33$, and then is displayed as a function of $\beta$. Error propagation via $\delta t_{c} \approx \delta \beta_{c} N_{\tau}\left(4 \pi^{2} / 33\right)$ was used. It has become customary, as in case for the Sommer scale $r_{0}$, to interpolate logarithmized QCD scales $\ln (s / a)$ with a rational Ansatz [21]

$$
\ln \left(\frac{s}{a}\right)=\left[\frac{\beta}{12 b_{0}}+\frac{b_{1}}{2 b_{0}^{2}} \ln \left(\frac{6 b_{0}}{\beta}\right)\right]\left(\frac{1+c_{1} \beta^{-1}+c_{2} \beta^{-2}}{1+c_{3} \beta^{-1}+c_{4} \beta^{-2}}\right)
$$

at values $b_{0}=11 /(4 \pi)^{2}$ and $b_{1}=102 /(4 \pi)^{4}$. We find the fit parameter values

$$
c_{1}=-9.5347(2060) \quad c_{2}=22.252(1.053) \quad c_{3}=-6.5581(2958) \quad c_{4}=6.547(1.241)
$$

at a reduced $\chi_{\text {d.o.f }}^{2}=0.87$ for the fit if all data points with $N_{\tau} \geq 6$ are included. See the curve in Fig. 2.

The finite size method for $\beta_{c}$ splits the phase space of the complex Polyakov loop into an outer region (Deconfinement phase) and an inner (Confined phase) and determines the coupling $\beta$ at which point the weight of the phases is 3 to 1 , because of $S U(3)$ 's $Z(3)$ center symmetry. A proper defined step function of weights $w_{c}$ and $w_{d}$ with $s(\beta)=\left(3 w_{c}-w_{d}\right) /\left(3 w_{c}+w_{d}\right)$ see Fig. 3, then has a zero which is determined by a linear fit. Our finite size determinations are all done with boxes $N_{s}>3 N_{\tau}$ except for $N_{\tau}=22$ at $N_{s}=64$. For values $N_{\tau} \leq 16$ we have checked, that the weight ratio method results are consistent with polynomial finite size scaling extrapolations of susceptibility peak positions at the first order phase transition. The weight ratio method however, and already for $N_{s} \geq 3 N_{\tau}$, has smaller corrections, which only are exponentially small in the spacial size. Our $\beta_{c}$ data in Table 2 exert stress on semi-analytic calculations $[22,23]$ but are consistent with the Bielefeld numbers [24, 25] as well as with Berg et. al. [26]. Deviations mostly lie within the $1 \sigma$ error margin.

In finite temperature lattice calculations, it is wishful to present the critical temperature $T_{\mathrm{c}}$ in physical units. If there are no precise measurements of physical observables like e.g. the string tension we can also opt for less physical zero temperature lattice scales. We determine two scales: the Sommer scale [8] denoted by $r_{0} / a$ and the Wilson flow $\sqrt{t_{0}} / a$ as recently introduced by Lüscher 


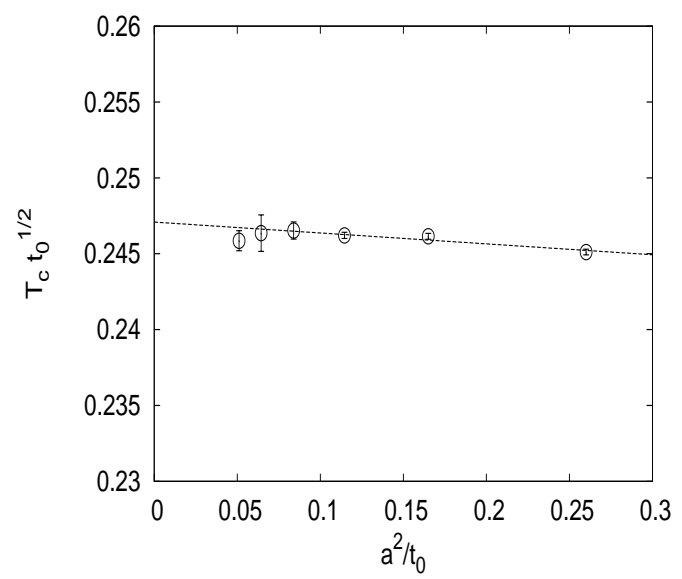

Figure 4: $T_{c} \sqrt{t_{0}}$ calculated from data in Table 2 at $N_{\tau}=8, \ldots, 18$ (data points from the right to the left). The $t_{0}$ data of [10] are used to interpolate $t_{0}$ by the rational form of eq. 3.1 .

$[9,10]$ and leave the presentation to forthcoming work [6]. In case of $t_{0}$ and for any given $\beta$, the lattice configuration is "cooled" using Runge Kutta algorithm for a classical Wilson flow until a stopping criterion on the energy density is reached at pseudo time $t_{0}$. Various different regularization's (Wilson, Wilson improved, Clover) for the energy density can be employed. Here we will use Lüscher's $t_{0}$ numbers from [10] for the range $5.96 \leq \beta \leq 6.59$, corresponding to the $N_{\tau}$ range $8 \leq N_{\tau} \leq 18$. We interpolate $\ln \left(\sqrt{t_{0}} / a\right)$ similar to $\ln (s / a)$ as in eq. 3.1 by a rational form and determine $T_{c} \sqrt{t_{0}}$ in the continuum limit $a^{2} / t_{0} \rightarrow 0$. We display in Fig. 4 the continuum extrapolation for $T_{c} \sqrt{t_{0}}$ based on the Lüscher data. A straight line fit, the line in Fig. 4, through all six data points with order $\mathscr{O}\left(a^{2}\right)$ corrections only, yields:

$$
T_{c} \sqrt{t_{0}}=0.2471(3)[10] \quad \chi_{\text {d.o.f }}^{2}=0.94 \text {. }
$$

We observe very small $a^{2}$ corrections of relative magnitude 0.004 accompanied by a small statistical error 0.0003 . Varying the fit range, and also adding $a^{4}$ corrections to the fit, we estimate an systematic uncertainty of 0.0010 in the observable and thus $T_{c} \sqrt{t_{0}}=0.2471(13)$ is a realistic finding. It is to be remarked, that the scaling deviations in the amplitude product turn out to be quite smooth and regular. Thus, under the provision of correct and precise $t_{0}$ data ${ }^{1}$, our $T_{c}$ determinations for $N_{\tau} \leq 18$ appear to be of similar numerical quality. Naturally the physical temperature is more relevant than $t_{0}$, unless $t_{0}$ can be converted into another physical scale at same precision levels.

\section{Conclusion}

We have estimated the critical couplings $\beta_{c}$ for the Wilson action and for temporal extents up to $N_{\tau}=20$ with relative precision levels $\leq 0.001$. For the $N_{\tau}$ interval $6, \ldots, 22$ we also provide a rational interpolation to $\ln \left(t_{c} / a\right)$ in eq. 3.2 and eq. 3.3, on the same footing as for the Sommer scale in [21], $t_{c}$ being the critical time extent. It remains to be seen how the determination of the diffusion

\footnotetext{
${ }^{1}$ The values of $t_{0} / a^{2}$ in [10] are probably affected by downward finite size effects of the order few percent, as the authors concede.
} 
constant $\kappa$ in eq. 2.6 responds to more elaborate models for the spectral function and depends on the finite quark mass and on the continuum limit. Work in this direction is in progress

Acknowledgements: Simulations where performed on the JARA0039 and JARA0108 accounts of JARA-HPC in Aachen, JUDGE/JUROPA at JSC Jülich, the OCuLUS Cluster in Paderborn, and the Bielefeld GPU cluster.

\section{References}

[1] H.B. Meyer, Eur. Phys. J. A 47 (2011) 86.

[2] G. Cuniberti, E. De Micheli and G.A. Viano, Commun. Math. Phys. 216 (2001) 59.

[3] Y. Burnier, M. Laine and L. Mether, Eur. Phys. J. C 71 (2011) 1619.

[4] A. Francis, O. Kaczmarek, M. Laine, M. Müller, T. Neuhaus and H. Ohno, Towards the continuum limit in transport coefficient computations, PoS (Lattice 2013) 453 [1311.3759].

[5] O. Kaczmarek, Quark Matter 2014 proceedings, ARXIV:1407.5918.

[6] A. Francis, O. Kaczmarek, M. Laine, T. Neuhaus and H. Ohno, Critical point and scale setting in $S U(3)$ plasma: an update, in preparation.

[7] A. Francis, O. Kaczmarek, M. Laine, T. Neuhaus and H. Ohno, A non-perturbative estimate of the heavy quark momentum diffusion coefficient, in preparation.

[8] R. Sommer, Nucl. Phys. B 411 (1994) 839.

[9] M. Lüscher, JHEP 08 (2010) 071.

[10] M. Lüscher and S. Schaefer, JHEP 07 (2011) 36.

[11] S. Caron-Huot, M. Laine and G.D. Moore, JHEP 04 (2009) 053.

[12] J. Casalderrey-Solana and D. Teaney, Phys. Rev. D 74 (2006) 085012.

[13] A. Francis, O. Kaczmarek, M. Laine and J. Langelage, Proceedings of the XXIX International Symposium on Lattice Field Theory (Lattice 2011), July 10-16, 2011. Squaw Valley, Lake Tahoe, California.

[14] M. Lüscher and P. Weisz, JHEP 09 (2001) 10.

[15] H.B. Meyer, Phys. Rev. D 76 (2007) 101701.

[16] G. Parisi, R. Petronzio and F. Rapuano, Phys. Lett. B 128 (1983) 418.

[17] P. de Forcrand and C. Roiesnel, Phys. Lett. B 151 (1985) 77.

[18] A. Francis, O. Kaczmarek, M. Laine and J. Langelage, PoS LATTICE 2011 (2011) 202.

[19] Y. Burnier, M. Laine, J. Langelage and L. Mether, JHEP 08 (2010) 094.

[20] C. Borgs, W. Janke, Phys. Rev. Lett. (68) (1992) 1738.

[21] S. Dürr, Z. Fodor, C. Hoelbling, T. Kurth, JHEP (0704) (2007) 55.

[22] J. Langelage et al, JHEP 02 (2011) 057 [Erratum-ibid. 07 (2011) 014] [1010.0951].

[23] X. Cheng and E. T. Tomboulis, Phys. Rev. D 86 (2012) 074507 [1206.3816].

[24] G. Boyd, J. Engels, F. Karsch, E. Laermann, C. Legeland, M. Luetgemeier and B. Petersson, Nucl. Phys. B469 (1996) 419.

[25] B. Beinlich, F. Karsch, E. Laermann and A. Peikert, Eur. Phys. J. C 6 (1999) 133 [hep-lat/9707023].

[26] B. A. Berg and H. Wu, Phys. Rev. D 05 (2013) 074507. 